\title{
A nonlocal three-point inclusion problem of Langevin equation with two different fractional orders
}

Bashir Ahmad ${ }^{1 *}$, Juan J Nieto ${ }^{1,2}$ and Ahmed Alsaedi ${ }^{1}$

\author{
* Correspondence: \\ bashir_qau@yahoo.com \\ 'Department of Mathematics, \\ Faculty of Science, King Abdulaziz \\ University, P. O. Box 80203, Jeddah \\ 21589, Saudi Arabia \\ Full list of author information is \\ available at the end of the article
}

\begin{abstract}
This article studies the existence of solutions for a three-point inclusion problem of Langevin equation with two fractional orders. Our main tools of study include a nonlinear alternative of Leray-Schauder type, selection theorem due to Bressan and Colombo for lower semicontinuous multivalued maps, and a fixed point theorem for multivalued map due to Covitz and Nadler. An illustrative example is also presented. Mathematical Subject Classification 2000: 26A33; 34A12; 34A40.
\end{abstract}

Keywords: Langevin equation, fractional order, inclusions, nonlocal boundary conditions, fixed point theorems

\section{Introduction}

The study of fractional calculus has recently gained a great momentum and has emerged as an interesting and important field of research. The popularity of the subject can easily be witnessed by a huge number of articles and books published in the last few years. It is mainly due to the extensive application of fractional calculus in the mathematical modeling of physical, engineering and biological phenomena. Fractional differential equations appear naturally in a number of fields such as physics, chemistry, biology, economics, control theory, signal and image processing, biophysics, blood flow phenomena, aerodynamics, fitting of experimental data, etc. For recent development in theory and applications of fractional calculus, see the books [1-4]. Some results concerning the initial and boundary value problems of fractional equations and inclusions can be found in a series of articles [5-26] and the references therein.

Langevin equation is found to be an effective tool to describe the evolution of physical phenomena in fluctuating environments [27-29]. Various generalizations of Langevin equation have been proposed to describe dynamical processes in a fractal medium. One such generalization is the generalized Langevin equation [30-35] which incorporates the fractal and memory properties with a dissipative memory kernel into the Langevin equation. In another possible extension, ordinary derivative is replaced by a fractional derivative in the Langevin equation to yield the fractional Langevin equation [36-39]. Recently, Lim et al. [40] have studied a new type of Langevin equation with 
two different fractional orders. The solution to this new version of fractional Langevin equation gives a fractional Gaussian process parameterized by two indices, which provides a more flexible model for fractal processes as compared with the usual one characterized by a single index. In [41], Lim et al. discussed the fractional oscillator process with two indices. Recently, a Dirichlet boundary value problem for Langevin equation involving two fractional orders has been studied in [42]. Some more recent work on Langevin equation can be found in [43-46]. In a more recent article [47], the authors studied a nonlinear Langevin equation involving two fractional orders $\alpha \in(0,1]$ and $\beta$ $\in(1,2]$ with three-point boundary conditions.

Motivated by recent work on Langevin equation of fractional order, we study the following inclusion problem of Langevin equation of two fractional orders in different intervals with three-point boundary conditions

$$
\left\{\begin{aligned}
{ }^{c} D^{\beta}\left({ }^{c} D^{\alpha}+\lambda\right) x(t) & \in F(t, x(t)), \quad 0<t<1, \quad 0<\alpha \leq 1, \quad 1<\beta \leq 2, \\
x(0) & =0, \quad x(\eta)=0, \quad x(1)=0, \quad 0<\eta<1,
\end{aligned}\right.
$$

where ${ }^{c} D$ is the Caputo fractional derivative, $F:[0,1] \times \mathbb{R} \rightarrow \mathcal{P}(\mathbb{R})$ is a compact valued multivalued map, and $\mathcal{P}(\mathbb{R})$ is the family of all subsets of $\mathbb{R}$.

We present some existence results for the problem (1.1), when the right hand side is convex as well as nonconvex valued. The first result relies on the nonlinear alternative of Leray-Schauder type. In the second result, we shall combine the nonlinear alternative of Leray-Schauder type for single-valued maps with a selection theorem due to Bressan and Colombo for lower semicontinuous multivalued maps with nonempty closed and decomposable values. The third result, dealing with a nonconvex valued right hand side of the fractional inclusion in (1.1), employs a fixed point theorem for multivalued map due to Covitz and Nadler. Here we remark that the single-valued case of (1.1) was discussed in [47].

\section{Background material for multivalued analysis}

First of all, we recall some basic definitions on multi-valued maps $[48,49]$.

For a normed space $(X,\|\cdot\|)$, let $P_{\mathrm{cl}}(X)=\{Y \in \mathcal{P}(X): Y$ is closed $\}$, $P_{\mathrm{cp}}(X)=\{Y \in \mathcal{P}(X): Y$ is compact $\}, \quad P_{\mathrm{cp}}(X)=\{Y \in \mathcal{P}(X): Y$ is compact $\}, \quad$ and $P_{\mathrm{c}, c}(X)=\{Y \in \mathcal{P}(X): Y$ is compact and convex $\}$. A multi-valued map $G: X \rightarrow \mathcal{P}(X)$ is convex (closed) valued if $G(x)$ is convex (closed) for all $x \in X$. The map $G$ is bounded on bounded sets if $G(\mathbb{B})=\bigcup_{x \in \mathbb{B}} G(x)$ is bounded in $X$ for all $\mathbb{B} \in P_{b}(X)$ (i. e. $\left.\sup _{x \in \mathbb{B}}\{\sup \{|y|: y \in G(x)\}\}<\infty\right)$. G is called upper semi-continuous (u.s.c.) on $X$ if for each $x_{0} \in X$, the set $G\left(x_{0}\right)$ is a nonempty closed subset of $X$, and if for each open set $N$ of $X$ containing $G\left(x_{0}\right)$, there exists an open neighborhood $\mathcal{N}_{0}$ of $x_{0}$ such that $G\left(\mathcal{N}_{0}\right) \subseteq N$. $G$ is said to be completely continuous if $G(\mathbb{B})$ is relatively compact for every $\mathbb{B} \in P_{b}(X)$. If the multi-valued map $G$ is completely continuous with nonempty compact values, then $G$ is u.s.c. if and only if $G$ has a closed graph, i.e., $x_{n} \rightarrow x_{*}, y_{n} \rightarrow$ $y_{*}, y_{n} \in G\left(x_{n}\right)$ imply $y_{*} \in G\left(x_{*}\right)$. G has a fixed point if there is $x \in X$ such that $x \in G$ $(x)$. The fixed point set of the multivalued operator $G$ will be denoted by Fix $G$. A multivalued map $G:[0 ; 1] \rightarrow P_{\mathrm{cl}}(\mathbb{R})$ is said to be measurable if for every $y \in \mathbb{R}$, the 
function

$$
t \mapsto d(y, G(t))=\inf \{|y-z|: z \in G(t)\}
$$

is measurable.

Definition 2.1 A multivalued map $F:[0,1] \times \mathbb{R} \rightarrow \mathcal{P}(\mathbb{R})$ is said to be Carathéodory if

(i) $t \rightarrow F(t, x)$ is measurable for each $x \in \mathbb{R}$;

(ii) $x \rightarrow F(t, x)$ is upper semicontinuous for almost all $t \in[0,1]$.

Further a Carathéodory function $F$ is called $L^{1}$-Carathéodory if

(iii) for each $\rho>0$, there exists $\phi_{\rho} \in L^{1}\left([0,1], \mathbb{R}^{+}\right)$such that

$$
\|F(t, x)\|=\sup \{|v|: v \in F(t, x)\} \leq \varphi_{\rho}(t)
$$

for all $\|x\|_{\infty} \leq \rho$ and for a.e. $t \in[0,1]$.

For each $y \in C([0,1], \mathbb{R})$, define the set of selections of $F$ by

$$
S_{F, y}:=\left\{v \in L^{1}([0,1], \mathbb{R}): v(t) \in F(t, \gamma(t)) \text { for a.e. } t \in[0,1]\right\} .
$$

Let $X$ be a nonempty closed subset of a Banach space $E$ and $G: X \rightarrow \mathcal{P}(E)$ is a multivalued operator with nonempty closed values. $G$ is lower semi-continuous (l.s.c.) if the set $\{y \in X: G(y) \cap B=\varnothing\}$ is open for any open set $B$ in $E$. Let $A$ be a subset of $[0,1] \times \mathbb{R} . A$ is $\mathcal{L} \otimes \mathscr{B}$ measurable if $A$ belongs to the $\sigma$-algebra generated by all sets of the form $\mathcal{J} \times \mathcal{D}$, where $\mathcal{J}$ is Lebesgue measurable in [0,1] and $\mathcal{D}$ is Borel measurable in $\mathbb{R}$. A subset $\mathcal{A}$ of $L^{1}([0,1], \mathbb{R})$ is decomposable if for all $u, v \in \mathcal{A}$ and measurable $\mathcal{J} \subset[0,1]=J$, the function $u \chi \mathcal{J}+v \chi_{J-\mathcal{J}} \in \mathcal{A}$, where $\chi_{\mathcal{J}}$ stands for the characteristic function of $\mathcal{J}$.

Definition 2.2 Let $Y$ be a separable metric space and let $N: Y \rightarrow \mathcal{P}\left(L^{1}([0,1], \mathbb{R})\right)$ be a multivalued operator. We say $N$ has a property $(B C)$ if $N$ is l.s.c. and has nonempty closed and decomposable values.

Let $F:[0,1] \times \mathbb{R} \rightarrow \mathcal{P}(\mathbb{R})$ be a multivalued map with nonempty compact values. Define a multivalued operator $\mathcal{F}: C([0,1] \times \mathbb{R}) \rightarrow \mathcal{P}\left(L^{1}([0,1], \mathbb{R})\right)$ associated with $F$ as

$$
\mathcal{F}(x)=\left\{w \in L^{1}([0,1], \mathbb{R}): w(t) \in F(t, x(t)) \text { for a.e. } t \in[0,1]\right\},
$$

which is called the Nemytskii operator associated with $F$.

Definition 2.3 Let $F:[0,1] \times \mathbb{R} \rightarrow \mathcal{P}(\mathbb{R})$ be a multivalued function with nonempty compact values. We say $F$ is of l.s.c. type if its associated Nemytskii operator $\mathscr{F}$ is l.s.c. and has nonempty closed and decomposable values. 
Let $(X, d)$ be a metric space induced from the normed space $(X,\|\cdot\|)$. Consider $H_{d}: \mathcal{P}(X) \times \mathcal{P}(X) \rightarrow \mathbb{R} \cup\{\infty\}$ given by

$$
H_{d}(A, B)=\max \left\{\sup _{a \in A} d(a, B), \sup _{b \in B} d(A, b)\right\},
$$

where $d(A, b)=\inf _{a L}{ }_{A} d(a ; b)$ and $d(a, B)=\inf _{b l}{ }_{B} d(a ; b)$. Then $\left(P_{b, c l}(X), H_{d}\right)$ is a metric space and $\left(P_{\mathrm{cl}}(X), H_{d}\right)$ is a generalized metric space (see [50]).

Definition 2.4 A multivalued operator $N: X \rightarrow P_{\mathrm{cl}}(X)$ is called

(a) $\gamma$-Lipschitz if and only if there exists $\gamma>0$ such that

$$
H_{d}(N(x), N(y)) \leq \gamma d(x, y) \text { for each } x, y \in X
$$

(b) a contraction if and only if it is $\gamma$-Lipschitz with $\gamma<1$.

The following lemmas will be used in the sequel.

Lemma 2.1 [51]Let $X$ be a Banach space. Let $F:[0,1] \times \mathbb{R} \rightarrow P_{\mathrm{cp}, c}(X)$ be an $L^{1}$-Carathéodory multivalued map and let $\Theta$ be a linear continuous mapping from $L^{1}([0,1]$, $X)$ to $C([0,1], X)$, then the operator

$$
\Theta \circ S_{F}: C([0,1], X) \rightarrow P_{\mathrm{cp}, c}(C([0,1], X)), \quad x \mapsto\left(\Theta \circ S_{F}\right)(x)=\Theta\left(S_{F, x}\right)
$$

is a closed graph operator in $C([0,1], X) \times C([0,1], X)$.

In passing, we remark that if $\operatorname{dim} X<\infty$, then $S_{F}(x) \neq \varnothing$ for any $x(\cdot) \in C([0,1], X)$ with $F(\cdot, \cdot)$ as in Lemma 2.1 .

Lemma 2.2 (Nonlinear alternative for Kakutani maps [52]) Let E be a Banach space, $C$ a closed convex subset of $E, U$ an open subset of $C$ and $0 \in U$. Suppose that $F: \bar{U} \rightarrow \mathcal{P}_{c, \mathrm{cv}}(C)$ is a upper semicontinuous compact map; here $\mathcal{P}_{c, \mathrm{cv}}(C)$ denotes the family of nonempty, compact convex subsets of $C$. Then either

(i) F has a fixed point in $\bar{U}$, or

(ii) there is $a u \in \partial U$ and $\lambda \in(0,1)$ with $u \in \lambda F(u)$.

Lemma 2.3 [53]Let $Y$ be a separable metric space and let $N: Y \rightarrow \mathcal{P}\left(L^{1}([0,1], \mathbb{R})\right)$ be a multivalued operator satisfying the property $(B C)$. Then $N$ has a continuous selection, that is, there exists a continuous function (single-valued) $g$ $: Y \rightarrow L^{1}([0,1], \mathbb{R})$ such that $g(x) \in N(x)$ for every $x \in Y$.

Lemma 2.4 [54] Let $(X, d)$ be a complete metric space. If $N: X \rightarrow P_{\mathrm{cl}}(X)$ is a contraction, then $\operatorname{Fix} N \neq \varnothing$.

For some recent results on multivalued maps, we refer the reader to the articles $[55,56]$. 


\section{Existence results}

We are concerned with the existence of solutions for the problem (1.1) when the right hand side has convex as well as nonconvex values. Initially, we assume that $F$ is a compact and convex valued multivalued map.

Definition 3.1 [47]A function $x \in C([0,1], \mathbb{R})$ is said to be a solution of (1.1), if there exists a function $v L L^{1}([0,1], \mathbb{R})$ with $v(t) \in F(t, x(t))$ a.e. $t \in[0,1]$ and

$$
\begin{aligned}
x(t) & =\int_{0}^{t} \frac{(t-u)^{\alpha-1}}{\Gamma(\alpha)}\left(\int_{0}^{u} \frac{(u-s)^{\beta-1}}{\Gamma(\beta)} v(s) d s-\lambda x(u)\right) d u \\
& +\frac{t^{\alpha}(\eta-t)}{(1-\eta)}\left[\int_{0}^{1} \frac{(1-u)^{\alpha-1}}{\Gamma(\alpha)}\left(\int_{0}^{u} \frac{(u-s)^{\beta-1}}{\Gamma(\beta)} v(s) d s-\lambda x(u)\right) d u\right] \\
& -\frac{t^{\alpha}(1-t)}{(1-\eta) \eta^{\alpha}}\left[\int_{0}^{\eta} \frac{(\eta-u)^{\alpha-1}}{\Gamma(\alpha)}\left(\int_{0}^{u} \frac{(u-s)^{\beta-1}}{\Gamma(\beta)} v(s) d s-\lambda x(u)\right) d u\right] .
\end{aligned}
$$

Theorem 3.1 Suppose that

$\left(H_{1}\right)$ the map $F:[0,1] \times \mathbb{R} \rightarrow \mathcal{P}(\mathbb{R})$ is Carathéodory and has nonempty compact convex values;

$\left(H_{2}\right)$ there exist a continuous non-decreasing function $\psi:[0, \infty) \rightarrow(0, \infty)$ and a positive continuous function $p$ such that

$$
\|F(t, x)\|_{\mathcal{P}}:=\sup \{|v|: v \in F(t, x)\} \leq p(t) \psi\left(\|x\|_{\infty}\right)
$$

for each $(t, u) \in[0, T] \times \mathbb{R}$;

$\left(H_{3}\right)$ there exists a number $M>0$ such that

$$
\frac{\left[\Gamma(\alpha+1)-|\lambda|\left(1+A\left(1+\eta^{\alpha+1}\right)\right)\right] \Gamma(\alpha+\beta+1)\|x\|_{\infty}}{\Gamma(\alpha+1)\left[1+A\left(\eta^{\alpha+1}+\eta^{\beta}\right)\right]\|p\|_{\infty} \psi\left(\|x\|_{\infty}\right)}>1,
$$

with $|\lambda|<\frac{\Gamma(\alpha+1)}{\left(1+A\left(1+\eta^{\alpha+1}\right)\right)}$ and $A=\frac{(\alpha)^{\alpha}}{(1-\eta)(1+\alpha)^{1+\alpha}}$.

Then the problem (1.1) has at least one solution.

Proof. In view of Definition 3.1, the existence of solutions to (1.1) is equivalent to the existence of solutions to the integral inclusion

$$
\begin{aligned}
x(t) \in & \int_{0}^{t} \frac{(t-u)^{\alpha-1}}{\Gamma(\alpha)}\left(\int_{0}^{u} \frac{(u-s)^{\beta-1}}{\Gamma(\beta)} F(s, u(s)) d s-\lambda x(u)\right) d u \\
& +\frac{t^{\alpha}(\eta-t)}{(1-\eta)}\left[\int_{0}^{1} \frac{(1-u)^{\alpha-1}}{\Gamma(\alpha)}\left(\int_{0}^{u} \frac{(u-s)^{\beta-1}}{\Gamma(\beta)} F(s, u(s)) d s-\lambda x(u)\right) d u\right] \\
& -\frac{t^{\alpha}(1-t)}{(1-\eta) \eta^{\alpha}}\left[\int_{0}^{\eta} \frac{(\eta-u)^{\alpha-1}}{\Gamma(\alpha)}\left(\int_{0}^{u} \frac{(u-s)^{\beta-1}}{\Gamma(\beta)} F(s, u(s)) d s-\lambda x(u)\right) d u\right], \quad t \in[0,1] .
\end{aligned}
$$


Let us introduce the operator $N: C([0,1], \mathbb{R}) \rightarrow \mathcal{P}(C([0,1], \mathbb{R}))$ as

$$
N(x)=\left\{\begin{array}{l}
h \in C([0,1], \mathbb{R}): \\
h(t)=\left\{\begin{array}{l}
\int_{0}^{t} \frac{(t-u)^{\alpha-1}}{\Gamma(\alpha)}\left(\int_{0}^{u} \frac{(u-s)^{\beta-1}}{\Gamma(\beta)} v(s) d s-\lambda x(u)\right) d u \\
+\frac{t^{\alpha}(\eta-t)}{(1-\eta)}\left[\int_{0}^{1} \frac{(1-u)^{\alpha-1}}{\Gamma(\alpha)}\left(\int_{0}^{u} \frac{(u-s)^{\beta-1}}{\Gamma(\beta)} v(s) d s-\lambda x(u)\right) d u\right] \\
-\frac{t^{\alpha}(1-t)}{(1-\eta) \eta^{\alpha}}\left[\int_{0}^{\eta} \frac{(\eta-u)^{\alpha-1}}{\Gamma(\alpha)}\left(\int_{0}^{u} \frac{(u-s)^{\beta-1}}{\Gamma(\beta)} v(s) d s-\lambda x(u)\right) d u\right],
\end{array}\right\}
\end{array}\right.
$$

for $v \in S_{F, x}$. We will show that the operator $N$ satisfies the assumptions of the nonlinear alternative of Leray-Schauder type. The proof consists of several steps. As a first step, we show that $N(x)$ is convex for each $x \in C([0,1], \mathbb{R})$. For that, let $h_{1}, h_{2} \in N(x)$. Then there exist $v_{1}, v_{2} \in S_{F, x}$ such that for each $t \in[0,1]$, we have

$$
\begin{aligned}
h_{i}(t)= & \int_{0}^{t} \frac{(t-u)^{\alpha-1}}{\Gamma(\alpha)}\left(\int_{0}^{u} \frac{(u-s)^{\beta-1}}{\Gamma(\beta)} v_{i}(s) d s-\lambda x(u)\right) d u \\
& +\frac{t^{\alpha}(\eta-t)}{(1-\eta)}\left[\int_{0}^{1} \frac{(1-u)^{\alpha-1}}{\Gamma(\alpha)}\left(\int_{0}^{u} \frac{(u-s)^{\beta-1}}{\Gamma(\beta)} v_{i}(s) d s-\lambda x(u)\right) d u\right] \\
& -\frac{t^{\alpha}(1-t)}{(1-\eta) \eta^{\alpha}}\left[\int_{0}^{\eta} \frac{(\eta-u)^{\alpha-1}}{\Gamma(\alpha)}\left(\int_{0}^{u} \frac{(u-s)^{\beta-1}}{\Gamma(\beta)} v_{i}(s) d s-\lambda x(u)\right) d u\right], \quad i=1,2 .
\end{aligned}
$$

Let $0 \leq \omega \leq 1$. Then, for each $t \in[0,1]$, we have

$$
\begin{aligned}
& {\left[\omega h_{1}+(1-\omega) h_{2}\right](t)} \\
& =\int_{0}^{t} \frac{(t-u)^{\alpha-1}}{\Gamma(\alpha)}\left(\int_{0}^{u} \frac{(u-s)^{\beta-1}}{\Gamma(\beta)}\left[\omega v_{1}(s)+(1-\omega) v_{2}(s)\right] d s-\lambda x(u)\right) d u \\
& +\frac{t^{\alpha}(\eta-t)}{(1-\eta)}\left[\int_{0}^{1} \frac{(1-u)^{\alpha-1}}{\Gamma(\alpha)}\left(\int_{0}^{u} \frac{(u-s)^{\beta-1}}{\Gamma(\beta)}\left[\omega v_{1}(s)+(1-\omega) v_{2}(s)\right] d s-\lambda x(u)\right) d u\right] \\
& -\frac{t^{\alpha}(1-t)}{(1-\eta) \eta^{\alpha}}\left[\int_{0}^{\eta} \frac{(\eta-u)^{\alpha-1}}{\Gamma(\alpha)}\left(\int_{0}^{u} \frac{(u-s)^{\beta-1}}{\Gamma(\beta)}\left[\omega v_{1}(s)+(1-\omega) v_{2}(s)\right] d s-\lambda x(u)\right) d u\right] .
\end{aligned}
$$

Since $S_{F, x}$ is convex ( $F$ has convex values), therefore it follows that $\omega h_{1}+(1-\omega) h_{2}$ $\in N(x)$.

Next, we show that $N(x)$ maps bounded sets into bounded sets in $C([0,1], \mathbb{R})$. For a positive number $r$, let $B_{r}=\left\{x \in C([0,1], \mathbb{R}):\|x\|_{\infty} \leq r\right\}$ be a bounded set in $C([0,1]$, $\mathbb{R})$. Then, for each $h \in N(x), x \in B_{r}$, there exists $v \in S_{F, x}$ such that 


$$
\begin{aligned}
h(t) & =\int_{0}^{t} \frac{(t-u)^{\alpha-1}}{\Gamma(\alpha)}\left(\int_{0}^{u} \frac{(u-s)^{\beta-1}}{\Gamma(\beta)} v(s) d s-\lambda x(u)\right) d u \\
& +\frac{t^{\alpha}(\eta-t)}{(1-\eta)}\left[\int_{0}^{1} \frac{(1-u)^{\alpha-1}}{\Gamma(\alpha)}\left(\int_{0}^{u} \frac{(u-s)^{\beta-1}}{\Gamma(\beta)} v(s) d s-\lambda x(u)\right) d u\right] \\
& -\frac{t^{\alpha}(1-t)}{(1-\eta) \eta^{\alpha}}\left[\int_{0}^{\eta} \frac{(\eta-u)^{\alpha-1}}{\Gamma(\alpha)}\left(\int_{0}^{u} \frac{(u-s)^{\beta-1}}{\Gamma(\beta)} v(s) d s-\lambda x(u)\right) d u\right],
\end{aligned}
$$

and

$$
\begin{aligned}
&|h(t)| \leq \sup _{t \in[0,1]}\left\{\int_{0}^{t} \frac{(t-u)^{\alpha-1}}{\Gamma(\alpha)}\left(\int_{0}^{u} \frac{(u-s)^{\beta-1}}{\Gamma(\beta)}|v(s)| d s+|\lambda|\|x(u)\|\right) d u\right\} \\
&+\sup _{t \in[0,1]}\left|\frac{t^{\alpha}(\eta-t)}{(1-\eta)}\right|\left[\int_{0}^{1} \frac{(1-u)^{\alpha-1}}{\Gamma(\alpha)}\left(\int_{0}^{u} \frac{(u-s)^{\beta-1}}{\Gamma(\beta)}|v(s)| d s+|\lambda|\|x(u)\|\right) d u\right] \\
&+\sup _{t \in[0,1]}\left|\frac{t^{\alpha}(1-t)}{(1-\eta) \eta^{\alpha}}\right|\left[\int_{0}^{\eta} \frac{(\eta-u)^{\alpha-1}}{\Gamma(\alpha)}\left(\int_{0}^{u} \frac{(u-s)^{\beta-1}}{\Gamma(\beta)}|v(s)| d s+|\lambda|\|x(u)\|\right) d u\right] \\
& \leq\|p\|_{\infty} \psi\left(\|x\|_{\infty}\right)\left(1+\frac{\eta(\alpha \eta)^{\alpha}}{(1-\eta)(1+\alpha)^{1+\alpha}}\right) \int_{0}^{1} \frac{(1-u)^{\alpha-1}}{\Gamma(\alpha)} \int_{0}^{u} \frac{(u-s)^{\beta-1}}{\Gamma(\beta)} d s d u \\
&+|\lambda|\|x\|_{\infty}\left(1+\frac{\eta(\alpha \eta)^{\alpha}}{(1-\eta)(1+\alpha)^{1+\alpha}}\right) \int_{0}^{1} \frac{(1-u)^{\alpha-1}}{\Gamma(\alpha)} d u \\
&+\left(\frac{\|p\|_{\infty} \psi\left(\|x\| \|_{\infty}\right)(\alpha)^{\alpha}}{(1-\eta) \eta^{\alpha}(1+\alpha)^{1+\alpha}}\right) \int_{0}^{\eta} \frac{(\eta-u)^{\alpha-1}}{\Gamma(\alpha)} \int_{0}^{u} \frac{(u-s)^{\beta-1}}{\Gamma(\beta)} d s d u \\
&+\left(\frac{|\lambda|\|x\|_{\infty}(\alpha)^{\alpha}}{(1-\eta) \eta^{\alpha}(1+\alpha)^{1+\alpha}}\right) \int_{0}^{\eta} \frac{(\eta-u)^{\alpha-1}}{\Gamma(\alpha)} d u,
\end{aligned}
$$

where $\|p\|_{\infty}=\sup _{t \in[0,1]} p(t)$. Using the relations for Beta function $B(.$, .):

$$
\begin{gathered}
B(\beta+1, \alpha)=\int_{0}^{1}(1-u)^{\alpha-1} u^{\beta} d u=\frac{\Gamma(\alpha) \Gamma(\beta+1)}{\Gamma(\alpha+\beta+1)}, \\
\int_{0}^{\eta}(\eta-u)^{\alpha-1} u^{\beta} d u=\frac{\Gamma(\alpha) \Gamma(\beta+1)}{\Gamma(\alpha+\beta+1)} \eta^{\alpha+\beta}
\end{gathered}
$$

we find that

$$
\|h(t)\|_{\infty} \leq \frac{\left[1+A\left(\eta^{\alpha+1}+\eta^{\beta}\right)\right]\|p\|_{\infty} \psi(r)}{\Gamma(\alpha+\beta+1)}+\frac{|\lambda|\left[1+A\left(1+\eta^{\alpha+1}\right)\right] r}{\Gamma(\alpha+1)}
$$

where

$$
A=\frac{(\alpha)^{\alpha}}{(1-\eta)(1+\alpha)^{1+\alpha}} .
$$

Now we show that $N$ maps bounded sets into equicontinuous sets of $C([0,1], \mathbb{R})$. Let $t^{\prime}, t^{\prime \prime} \in[0,1]$ with $t^{\prime}<t^{\prime \prime}$ and $x \in B_{r}$, where $B_{r}$ is a bounded set of $C([0,1], \mathbb{R})$. For each $h \in N(x)$, we obtain 


$$
\begin{aligned}
& \left|h\left(t^{\prime \prime}\right)-h\left(t^{\prime}\right)\right| \\
& =\mid \int_{0}^{t^{\prime \prime}} \frac{\left(t^{\prime \prime}-u\right)^{\alpha-1}}{\Gamma(\alpha)}\left(\int_{0}^{u} \frac{(u-s)^{\beta-1}}{\Gamma(\beta)}|v(s)| d s+\lambda|x(u)|\right) d u \\
& -\int_{0}^{t^{\prime}} \frac{\left(t^{\prime}-u\right)^{\alpha-1}}{\Gamma(\alpha)}\left(\int_{0}^{u} \frac{(u-s)^{\beta-1}}{\Gamma(\beta)}|v(s)| d s+\lambda|x(u)|\right) d u \\
& +\frac{\left[\left(\left(t^{\prime \prime}\right)^{\alpha}-\left(t^{\prime}\right)^{\alpha}\right) \eta-\left(\left(t^{\prime \prime}\right)^{\alpha+1}-\left(t^{\prime}\right)^{\alpha+1}\right)\right]}{(1-\eta)} \\
& \times\left[\int_{0}^{1} \frac{(1-u)^{\alpha-1}}{\Gamma(\alpha)}\left(\int_{0}^{u} \frac{(u-s)^{\beta-1}}{\Gamma(\beta)} v(s) d s-\lambda x(u)\right) d u\right] \\
& -\frac{\left[\left(\left(t^{\prime \prime}\right)^{\alpha}-\left(t^{\prime}\right)^{\alpha}\right)-\left(\left(t^{\prime \prime}\right)^{\alpha+1}-\left(t^{\prime}\right)^{\alpha+1}\right)\right]}{(1-\eta) \eta^{\alpha}} \\
& \times\left[\int_{0}^{\eta} \frac{(\eta-u)^{\alpha-1}}{\Gamma(\alpha)}\left(\int_{0}^{u} \frac{(u-s)^{\beta-1}}{\Gamma(\beta)} v(s) d s-\lambda x(u)\right) d u\right] \mid \\
& \leq \mid \int_{0}^{t^{\prime}} \frac{\left[\left(t^{\prime \prime}-u\right)^{\alpha-1}-\left(t^{\prime}-u\right)^{\alpha-1}\right]}{\Gamma(\alpha)}\left(\int_{0}^{u} \frac{(u-s)^{\beta-1}}{\Gamma(\beta)}|v(s)| d s+\lambda|x(u)|\right) d u \\
& +\int_{t^{\prime}}^{t^{\prime \prime}} \frac{\left(t^{\prime \prime}-u\right)^{\alpha-1}}{\Gamma(\alpha)}\left(\int_{0}^{u} \frac{(u-s)^{\beta-1}}{\Gamma(\beta)}|v(s)| d s+\lambda|x(u)|\right) d u \mid \\
& +\left|\frac{\left[\left(\left(t^{\prime \prime}\right)^{\alpha}-\left(t^{\prime}\right)^{\alpha}\right) \eta-\left(\left(t^{\prime \prime}\right)^{\alpha+1}-\left(t^{\prime}\right)^{\alpha+1}\right)\right]}{(1-\eta)}\right| \\
& \times\left[\int_{0}^{1} \frac{(1-u)^{\alpha-1}}{\Gamma(\alpha)}\left(\int_{0}^{u} \frac{(u-s)^{\beta-1}}{\Gamma(\beta)} v(s) d s-\lambda x(u)\right) d u\right] \\
& +\left|\frac{\left[\left(\left(t^{\prime \prime}\right)^{\alpha}-\left(t^{\prime}\right)^{\alpha}\right)-\left(\left(t^{\prime \prime}\right)^{\alpha+1}-\left(t^{\prime}\right)^{\alpha+1}\right)\right]}{(1-\eta) \eta^{\alpha}}\right| \\
& \times\left[\int_{0}^{\eta} \frac{(\eta-u)^{\alpha-1}}{\Gamma(\alpha)}\left(\int_{0}^{u} \frac{(u-s)^{\beta-1}}{\Gamma(\beta)} v(s) d s-\lambda x(u)\right) d u\right] \\
& \leq \mid \int_{0}^{t^{\prime}} \frac{\left[\left(t^{\prime \prime}-u\right)^{\alpha-1}-\left(t^{\prime}-u\right)^{\alpha-1}\right]}{\Gamma(\alpha)}\left(\left(\|p\|_{\infty} \psi\left(\|x\|_{\infty}\right) \int_{0}^{u} \frac{(u-s)^{\beta-1}}{\Gamma(\beta)} d s+\lambda|x(u)|\right) d u\right. \\
& +\int_{t^{\prime}}^{t^{\prime \prime}} \frac{\left(t^{\prime \prime}-u\right)^{\alpha-1}}{\Gamma(\alpha)}\left(\left(\|p\|_{\infty} \psi\left(\|x\|_{\infty}\right) \int_{0}^{u} \frac{(u-s)^{\beta-1}}{\Gamma(\beta)} d s+\lambda|x(u)|\right) d u \mid\right. \\
& +\mid \frac{\left[\left(\left(t^{\prime \prime}\right)^{\alpha}-\left(t^{\prime}\right)^{\alpha}\right) \eta-\left(\left(t^{\prime \prime}\right)^{\alpha+1}-\left(t^{\prime}\right)^{\alpha+1}\right)\right]}{(1-\eta)} \\
& \times\left[\int_{0}^{1} \frac{(1-u)^{\alpha-1}}{\Gamma(\alpha)}\left(\left(\|p\|_{\infty} \psi\left(\|x\|_{\infty}\right) \int_{0}^{u} \frac{(u-s)^{\beta-1}}{\Gamma(\beta)} d s-\lambda x(u)\right) d u\right] \mid\right. \\
& +\mid \frac{\left[\left(\left(t^{\prime \prime}\right)^{\alpha}-\left(t^{\prime}\right)^{\alpha}\right)-\left(\left(t^{\prime \prime}\right)^{\alpha+1}-\left(t^{\prime}\right)^{\alpha+1}\right)\right]}{(1-\eta) \eta^{\alpha}} \\
& \times \mid\left[\int_{0}^{\eta} \frac{(\eta-u)^{\alpha-1}}{\Gamma(\alpha)}\left(\left(\|p\|_{\infty} \psi\left(\|x\|_{\infty}\right) \int_{0}^{u} \frac{(u-s)^{\beta-1}}{\Gamma(\beta)} d s-\lambda x(u)\right) d u\right] .\right.
\end{aligned}
$$


Obviously the right hand side of the above inequality tends to zero independently of $x \in$ $B_{r}$ as $t^{\prime \prime}-t^{\prime} \rightarrow 0$. As $N$ satisfies the above three assumptions, therefore it follows by AscoliArzelá theorem that $N: C([0,1], \mathbb{R}) \rightarrow \mathcal{P}(C([0,1], \mathbb{R}))$ is completely continuous.

In our next step, we show that $N$ has a closed graph. Let $x_{n} \rightarrow x_{n}, h_{n} \in N\left(x_{n}\right)$ and $h_{n}$ $\rightarrow h^{*}$. Then we need to show that $h_{*} \in N\left(x_{*}\right)$. Associated with $h_{n} \in N\left(x_{n}\right)$, there exists $v_{n} \in S_{F, x_{n}}$ such that for each $t \in[0,1]$,

$$
\begin{aligned}
h_{n}(t) & =\int_{0}^{t} \frac{(t-u)^{\alpha-1}}{\Gamma(\alpha)}\left(\int_{0}^{u} \frac{(u-s)^{\beta-1}}{\Gamma(\beta)} v_{n}(s) d s-\lambda x_{n}(u)\right) d u \\
& +\frac{t^{\alpha}(\eta-t)}{(1-\eta)}\left[\int_{0}^{1} \frac{(1-u)^{\alpha-1}}{\Gamma(\alpha)}\left(\int_{0}^{u} \frac{(u-s)^{\beta-1}}{\Gamma(\beta)} v_{n}(s) d s-\lambda x_{n}(u)\right) d u\right] \\
& -\frac{t^{\alpha}(1-t)}{(1-\eta) \eta^{\alpha}}\left[\int_{0}^{\eta} \frac{(\eta-u)^{\alpha-1}}{\Gamma(\alpha)}\left(\int_{0}^{u} \frac{(u-s)^{\beta-1}}{\Gamma(\beta)} v_{n}(s) d s-\lambda x_{n}(u)\right) d u\right] .
\end{aligned}
$$

Thus we have to show that there exists $v_{*} \in S_{F, x_{*}}$ such that for each $t \in[0,1]$,

$$
\begin{aligned}
h_{*}(t) & =\int_{0}^{t} \frac{(t-u)^{\alpha-1}}{\Gamma(\alpha)}\left(\int_{0}^{u} \frac{(u-s)^{\beta-1}}{\Gamma(\beta)} v_{*}(s) d s-\lambda x_{*}(u)\right) d u \\
& +\frac{t^{\alpha}(\eta-t)}{(1-\eta)}\left[\int_{0}^{1} \frac{(1-u)^{\alpha-1}}{\Gamma(\alpha)}\left(\int_{0}^{u} \frac{(u-s)^{\beta-1}}{\Gamma(\beta)} v_{*}(s) d s-\lambda x_{*}(u)\right) d u\right] \\
& -\frac{t^{\alpha}(1-t)}{(1-\eta) \eta^{\alpha}}\left[\int_{0}^{\eta} \frac{(\eta-u)^{\alpha-1}}{\Gamma(\alpha)}\left(\int_{0}^{u} \frac{(u-s)^{\beta-1}}{\Gamma(\beta)} v_{*}(s) d s-\lambda x_{*}(u)\right) d u\right] .
\end{aligned}
$$

Let us consider the continuous linear operator $\Theta: L^{1}([0,1], \mathbb{R}) \rightarrow C([0,1], \mathbb{R})$ so that

$$
\begin{aligned}
v \mapsto \Theta(v) & =\int_{0}^{t} \frac{(t-u)^{\alpha-1}}{\Gamma(\alpha)}\left(\int_{0}^{u} \frac{(u-s)^{\beta-1}}{\Gamma(\beta)} v(s) d s-\lambda x(u)\right) d u \\
& +\frac{t^{\alpha}(\eta-t)}{(1-\eta)}\left[\int_{0}^{1} \frac{(1-u)^{\alpha-1}}{\Gamma(\alpha)}\left(\int_{0}^{u} \frac{(u-s)^{\beta-1}}{\Gamma(\beta)} v(s) d s-\lambda x(u)\right) d u\right] \\
& -\frac{t^{\alpha}(1-t)}{(1-\eta) \eta^{\alpha}}\left[\int_{0}^{\eta} \frac{(\eta-u)^{\alpha-1}}{\Gamma(\alpha)}\left(\int_{0}^{u} \frac{(u-s)^{\beta-1}}{\Gamma(\beta)} v(s) d s-\lambda x(u)\right) d u\right] .
\end{aligned}
$$

Observe that

$$
\begin{aligned}
& \left\|h_{n}(t)-h_{*}(t)\right\| \\
& =\| \int_{0}^{t} \frac{(t-u)^{\alpha-1}}{\Gamma(\alpha)}\left(\int_{0}^{u} \frac{(u-s)^{\beta-1}}{\Gamma(\beta)}\left(v_{n}(s)-v_{*}(s)\right) d s-\lambda\left(x_{n}(u)-x_{*}(u)\right)\right) d u \\
& +\frac{t^{\alpha}(\eta-t)}{(1-\eta)}\left[\int_{0}^{1} \frac{(1-u)^{\alpha-1}}{\Gamma(\alpha)}\left(\int_{0}^{u} \frac{(u-s)^{\beta-1}}{\Gamma(\beta)}\left(v_{n}(s)-v_{*}(s)\right) d s-\lambda\left(x_{n}(u)-x_{*}(u)\right)\right) d u\right] \\
& -\frac{t^{\alpha}(1-t)}{(1-\eta) \eta^{\alpha}}\left[\int_{0}^{\eta} \frac{(\eta-u)^{\alpha-1}}{\Gamma(\alpha)}\left(\int_{0}^{u} \frac{(u-s)^{\beta-1}}{\Gamma(\beta)}\left(v_{n}(s)-v_{*}(s)\right) d s-\lambda\left(x_{n}(u)-x_{*}(u)\right)\right) d u\right] \| \\
& \rightarrow 0 \text { as } n \rightarrow \infty .
\end{aligned}
$$


Thus, it follows by Lemma 2.1 that $\Theta \circ S_{F}$ is a closed graph operator. Further, we have $h_{n}(t) \in \Theta\left(S_{F, x_{n}}\right)$. Since $x_{n} \rightarrow x_{n}$, it follows that

$$
\begin{aligned}
h_{*}(t) & =\int_{0}^{t} \frac{(t-u)^{\alpha-1}}{\Gamma(\alpha)}\left(\int_{0}^{u} \frac{(u-s)^{\beta-1}}{\Gamma(\beta)} v_{*}(s) d s-\lambda x_{*}(u)\right) d u \\
& +\frac{t^{\alpha}(\eta-t)}{(1-\eta)}\left[\int_{0}^{1} \frac{(1-u)^{\alpha-1}}{\Gamma(\alpha)}\left(\int_{0}^{u} \frac{(u-s)^{\beta-1}}{\Gamma(\beta)} v_{*}(s) d s-\lambda x_{*}(u)\right) d u\right] \\
& -\frac{t^{\alpha}(1-t)}{(1-\eta) \eta^{\alpha}}\left[\int_{0}^{\eta} \frac{(\eta-u)^{\alpha-1}}{\Gamma(\alpha)}\left(\int_{0}^{u} \frac{(u-s)^{\beta-1}}{\Gamma(\beta)} v_{*}(s) d s-\lambda x_{*}(u)\right) d u\right],
\end{aligned}
$$

for some $v_{*} \in S_{F, x_{*}}$.

Finally, we discuss a priori bounds on solutions. Let $x$ be a solution of (1.1). Then there exists $v \in L^{1}([0,1], \mathbb{R})$ with $v \in S_{F, x}$ such that, for $t \in[0,1]$, we have

$$
\begin{aligned}
x(t) & =\int_{0}^{t} \frac{(t-u)^{\alpha-1}}{\Gamma(\alpha)}\left(\int_{0}^{u} \frac{(u-s)^{\beta-1}}{\Gamma(\beta)} f(s) d s-\lambda x(u)\right) d u \\
& +\frac{t^{\alpha}(\eta-t)}{(1-\eta)}\left[\int_{0}^{1} \frac{(1-u)^{\alpha-1}}{\Gamma(\alpha)}\left(\int_{0}^{u} \frac{(u-s)^{\beta-1}}{\Gamma(\beta)} f(s) d s-\lambda x(u)\right) d u\right] \\
& -\frac{t^{\alpha}(1-t)}{(1-\eta) \eta^{\alpha}}\left[\int_{0}^{\eta} \frac{(\eta-u)^{\alpha-1}}{\Gamma(\alpha)}\left(\int_{0}^{u} \frac{(u-s)^{\beta-1}}{\Gamma(\beta)} f(s) d s-\lambda x(u)\right) d u\right] .
\end{aligned}
$$

In view of $\left(H_{2}\right)$ together with the condition $|\lambda|<\frac{\Gamma(\alpha+1)}{\left(1+A\left(1+\eta^{\alpha+1}\right)\right)}$, for each $t \in[0,1]$, we find that

$$
\|x\|_{\infty} \leq \frac{\Gamma(\alpha+1)\left[1+A\left(\eta^{\alpha+1}+\eta^{\beta}\right)\right]\|p\|_{\infty} \psi\left(\|x\|_{\infty}\right)}{\left[\Gamma(\alpha+1)-|\lambda|\left(1+A\left(1+\eta^{\alpha+1}\right)\right)\right] \Gamma(\alpha+\beta+1)}
$$

which can alternatively be written as

$$
\frac{\left[\Gamma(\alpha+1)-|\lambda|\left(1+A\left(1+\eta^{\alpha+1}\right)\right)\right] \Gamma(\alpha+\beta+1)\|x\|_{\infty}}{\Gamma(\alpha+1)\left[1+A\left(\eta^{\alpha+1}+\eta^{\beta}\right)\right]\|p\|_{\infty} \psi\left(\|x\|_{\infty}\right)} \leq 1,
$$

In view of $\left(H_{3}\right)$, there exists $M$ such that $\|x\|_{\infty} \neq M$. Let us set

$$
U=\left\{x \in C([0,1], \mathbb{R}):\|x\|_{\infty}<M+1\right\} .
$$

Note that the operator is $N: \bar{U} \rightarrow \mathcal{P}(C([0,1], \mathbb{R}))$ is upper semicontinuous and completely continuous. From the choice of $U$, there is no $x \in \partial U$ such that $x \in \mu N(x)$ for some $\mu \in(0,1)$. Consequently, by the nonlinear alternative of Leray-Schauder type (Lemma 2.2), we deduce that $N$ has a fixed point $x \in \bar{U}$ which is a solution of the problem (1.1). This completes the proof. $\square$

Next, we study the case where $F$ is not necessarily convex valued. Our approach here is based on the nonlinear alternative of Leray-Schauder type combined with the selection theorem of Bressan and Colombo for l.s.c. maps with decomposable values. 
Theorem 3.2 Assume that $\left(H_{2}\right)-\left(H_{3}\right)$ and the following conditions hold:

$\left(H_{4}\right) F:[0,1] \times \mathbb{R} \rightarrow \mathcal{P}(\mathbb{R})$ is a nonempty compact-valued multivalued map such that

(a) $(t, x) \mapsto F(t, x)$ is $\mathcal{L} \otimes \mathscr{B}$ measurable,

(b) $x \mapsto F(t, x)$ is lower semicontinuous for each $t \in[0,1]$;

$\left(H_{5}\right)$ for each $\sigma>0$, there exists $\phi_{\sigma} \in C\left([0,1], \mathbb{R}_{+}\right)$such that

$$
\|F(t, x)\|=\sup \{|y|: y \in F(t, x)\} \leq \varphi_{\sigma}(t) \text { for all }\|x\|_{\infty} \leq \sigma \text { and for a.e.t } \in[0,1] .
$$

Then the boundary value problem (1.1) has at least one solution on $[0,1]$.

Proof. It follows from $\left(H_{4}\right)$ and $\left(H_{5}\right)$ that $F$ is of l.s.c. type. Then from Lemma 2.3, there exists a continuous function $f: C([0,1], \mathbb{R}) \rightarrow L^{1}([0,1], \mathbb{R})$ such that $f(x) \in \mathscr{F}(x)$ for all $x L C([0,1], \mathbb{R})$.

Consider the problem

$$
\left\{\begin{array}{c}
{ }^{c} D^{\beta}\left({ }^{c} D^{\alpha}+\lambda\right) x(t)=f(x(t)), \quad 0<t<1, \quad 0<\alpha \leq 1, \quad 1<\beta \leq 2, \\
x(0)=0, \quad x(\eta)=0, \quad x(1)=0, \quad 0<\eta<1,
\end{array}\right.
$$

Observe that if $x \in C^{3}([0,1])$ is a solution of (3.2), then $x$ is a solution to the problem (1.1). In order to transform the problem (3.2) into a fixed point problem, we define the operator $\bar{N}$ as

$$
\begin{aligned}
(\bar{N} x)(t) & =\int_{0}^{t} \frac{(t-u)^{\alpha-1}}{\Gamma(\alpha)}\left(\int_{0}^{u} \frac{(u-s)^{\beta-1}}{\Gamma(\beta)} f(x(s)) d s-\lambda x(u)\right) d u \\
& +\frac{t^{\alpha}(\eta-t)}{(1-\eta)}\left[\int_{0}^{1} \frac{(1-u)^{\alpha-1}}{\Gamma(\alpha)}\left(\int_{0}^{u} \frac{(u-s)^{\beta-1}}{\Gamma(\beta)} f(x(s)) d s-\lambda x(u)\right) d u\right] \\
& -\frac{t^{\alpha}(1-t)}{(1-\eta) \eta^{\alpha}}\left[\int_{0}^{\eta} \frac{(\eta-u)^{\alpha-1}}{\Gamma(\alpha)}\left(\int_{0}^{u} \frac{(u-s)^{\beta-1}}{\Gamma(\beta)} f(x(s)) d s-\lambda x(u)\right) d u\right] .
\end{aligned}
$$

It can easily be shown that $\bar{N}$ is continuous and completely continuous. The remaining part of the proof is similar to that of Theorem 3.1. So we omit it. This completes the proof.

口

Now we prove the existence of solutions for the problem (1.1) with a nonconvex valued right hand side by applying a fixed point theorem for multivalued map due to Covitz and Nadler (Lemma 2.4).

Theorem 3.3 Assume that the following conditions hold:

$\left(H_{6}\right) F:[0,1] \times \mathbb{R} \rightarrow P_{\mathrm{cp}}(\mathbb{R})$ is such that $F(, x):[0,1] \rightarrow P_{\mathrm{cp}}(\mathbb{R})$ is measurable for each $x \in \mathbb{R}$;

$\left(H_{7}\right) H_{d}(F(t, x), F(t, \bar{x})) \leq m(t)|x-\bar{x}|$ for almost all $t \in[0,1]$ and $x, \bar{x} \in \mathbb{R}$ with $m \in$ $C\left([0,1], \mathbb{R}^{+}\right)$and $d(0, F(t, 0)) \leq m(t)$ for almost all $t \in[0,1]$. 
Then the boundary value problem (1.1) has at least one solution on $[0,1]$ if

$$
\left[\frac{\|m\|_{\infty}}{\Gamma(\alpha+\beta+1)}\left(1+a\left(\eta^{\alpha+1}+\eta^{\beta}\right)\right)+\frac{|\lambda|}{\Gamma(\alpha+1)}\left(1+a\left(1+\eta^{\alpha+1}\right)\right)\right]<1 .
$$

Proof. Observe that the set $S_{F, x}$ is nonempty for each $x \in C([0,1], \mathbb{R})$ by the assumption $\left(H_{6}\right)$, so $F$ has a measurable selection (see [[57], Theorem III.6]). Now we show that the operator $N$ defined by (3.1) satisfies the assumptions of Lemma 11.2.4. To show that $N(x) \in P_{\mathrm{cl}}((C[0,1], \mathbb{R}))$ for each $x \in C([0,1], \mathbb{R})$, let $\left\{u_{n}\right\}_{n} \geq 0 \in N(x)$ be such that $u_{n} \rightarrow u(n \rightarrow \infty)$ in $C([0,1], \mathbb{R})$. Then $u \in C([0,1], \mathbb{R})$ and there exists $v_{n} \in$ $S_{F, x}$ such that, for each $t \in[0,1]$,

$$
\begin{aligned}
u_{n}(t) & =\int_{0}^{t} \frac{(t-u)^{\alpha-1}}{\Gamma(\alpha)}\left(\int_{0}^{u} \frac{(u-s)^{\beta-1}}{\Gamma(\beta)} v_{n}(s) d s-\lambda x(u)\right) d u \\
& +\frac{t^{\alpha}(\eta-t)}{(1-\eta)}\left[\int_{0}^{1} \frac{(1-u)^{\alpha-1}}{\Gamma(\alpha)}\left(\int_{0}^{u} \frac{(u-s)^{\beta-1}}{\Gamma(\beta)} v_{n}(s) d s-\lambda x(u)\right) d u\right] \\
& -\frac{t^{\alpha}(1-t)}{(1-\eta) \eta^{\alpha}}\left[\int_{0}^{\eta} \frac{(\eta-u)^{\alpha-1}}{\Gamma(\alpha)}\left(\int_{0}^{u} \frac{(u-s)^{\beta-1}}{\Gamma(\beta)} v_{n}(s) d s-\lambda x(u)\right) d u\right] .
\end{aligned}
$$

As $F$ has compact values, we pass onto a subsequence to obtain that $v_{n}$ converges to $v$ in $L^{1}([0,1], \mathbb{R})$. Thus, $v \in S_{F, x}$ and for each $t \in[0,1]$,

$$
\begin{aligned}
u_{n}(t) \rightarrow u(t) & =\int_{0}^{t} \frac{(t-u)^{\alpha-1}}{\Gamma(\alpha)}\left(\int_{0}^{u} \frac{(u-s)^{\beta-1}}{\Gamma(\beta)} v(s) d s-\lambda x(u)\right) d u \\
& +\frac{t^{\alpha}(\eta-t)}{(1-\eta)}\left[\int_{0}^{1} \frac{(1-u)^{\alpha-1}}{\Gamma(\alpha)}\left(\int_{0}^{u} \frac{(u-s)^{\beta-1}}{\Gamma(\beta)} v(s) d s-\lambda x(u)\right) d u\right] \\
& -\frac{t^{\alpha}(1-t)}{(1-\eta) \eta^{\alpha}}\left[\int_{0}^{\eta} \frac{(\eta-u)^{\alpha-1}}{\Gamma(\alpha)}\left(\int_{0}^{u} \frac{(u-s)^{\beta-1}}{\Gamma(\beta)} v(s) d s-\lambda x(u)\right) d u\right] .
\end{aligned}
$$

Hence, $u \in N(x)$.

Next we show that there exists $\gamma<1$ such that

$$
H_{d}(N(x), N(\bar{x})) \leq \gamma\|x-\bar{x}\|_{\infty} \quad \text { for each } \quad x, \bar{x} \in C([0,1], \mathbb{R}) .
$$

Let $x, \bar{x} \in C([0,1], \mathbb{R})$ and $h_{1} \in N(x)$. Then there exists $v_{1}(t) \in F(t, x(t))$ such that, for each $t \in[0,1]$,

$$
\begin{aligned}
h_{1}(t) & =\int_{0}^{t} \frac{(t-u)^{\alpha-1}}{\Gamma(\alpha)}\left(\int_{0}^{u} \frac{(u-s)^{\beta-1}}{\Gamma(\beta)} v_{1}(s) d s-\lambda x(u)\right) d u \\
& +\frac{t^{\alpha}(\eta-t)}{(1-\eta)}\left[\int_{0}^{1} \frac{(1-u)^{\alpha-1}}{\Gamma(\alpha)}\left(\int_{0}^{u} \frac{(u-s)^{\beta-1}}{\Gamma(\beta)} v_{1}(s) d s-\lambda x(u)\right) d u\right] \\
& -\frac{t^{\alpha}(1-t)}{(1-\eta) \eta^{\alpha}}\left[\int_{0}^{\eta} \frac{(\eta-u)^{\alpha-1}}{\Gamma(\alpha)}\left(\int_{0}^{u} \frac{(u-s)^{\beta-1}}{\Gamma(\beta)} v_{1}(s) d s-\lambda x(u)\right) d u\right] .
\end{aligned}
$$


By $\left(H_{7}\right)$, we have

$$
H_{d}(F(t, x), F(t, \bar{x})) \leq m(t)|x(t)-\bar{x}(t)| .
$$

So, there exists $w \in F(t, \bar{x}(t))$ such that

$$
\left|v_{1}(t)-w\right| \leq m(t)|x(t)-\bar{x}(t)|, \quad t \in[0,1] .
$$

Define $U:[0,1] \rightarrow \mathcal{P}(\mathbb{R})$ by

$$
U(t)=\left\{w \in \mathbb{R}:\left|v_{1}(t)-w\right| \leq m(t)|x(t)-\bar{x}(t)|\right\} .
$$

Since the multivalued operator $U(t) \cap F(t, \bar{x}(t))$ is measurable ([[57], Proposition III.4])), there exists a function $v_{2}(t)$ which is a measurable selection for $V$. So $v_{2}(t) \in F(t, \bar{x}(t))$ and for each $t \in[0,1]$, we have $\left|v_{1}(t)-v_{2}(t)\right| \leq m(t)|x(t)-\bar{x}(t)|$.

For each $t \in[0,1]$, let us define

$$
\begin{aligned}
h_{2}(t) & =\int_{0}^{t} \frac{(t-u)^{\alpha-1}}{\Gamma(\alpha)}\left(\int_{0}^{u} \frac{(u-s)^{\beta-1}}{\Gamma(\beta)} v_{2}(s) d s-\lambda \bar{x}(u)\right) d u \\
& +\frac{t^{\alpha}(\eta-t)}{(1-\eta)}\left[\int_{0}^{1} \frac{(1-u)^{\alpha-1}}{\Gamma(\alpha)}\left(\int_{0}^{u} \frac{(u-s)^{\beta-1}}{\Gamma(\beta)} v_{2}(s) d s-\lambda \bar{x}(u)\right) d u\right] \\
& -\frac{t^{\alpha}(1-t)}{(1-\eta) \eta^{\alpha}}\left[\int_{0}^{\eta} \frac{(\eta-u)^{\alpha-1}}{\Gamma(\alpha)}\left(\int_{0}^{u} \frac{(u-s)^{\beta-1}}{\Gamma(\beta)} v_{2}(s) d s-\lambda \bar{x}(u)\right) d u\right] .
\end{aligned}
$$

Thus,

$$
\begin{aligned}
& \left|h_{1}(t)-h_{2}(t)\right| \\
\leq & \int_{0}^{t} \frac{(t-u)^{\alpha-1}}{\Gamma(\alpha)}\left(\int_{0}^{u} \frac{(u-s)^{\beta-1}}{\Gamma(\beta)}\left|v_{1}(s)-v_{2}(s)\right| d s+|\lambda||x(u)-\bar{x}(u)|\right) d u \\
+ & \left|\frac{t^{\alpha}(\eta-t)}{(1-\eta)}\right|\left[\int_{0}^{1} \frac{(1-u)^{\alpha-1}}{\Gamma(\alpha)}\left(\int_{0}^{u} \frac{(u-s)^{\beta-1}}{\Gamma(\beta)}\left|v_{1}(s)-v_{2}(s)\right| d s|+\lambda||x(u)-\bar{x}(u)|\right) d u\right] \\
+ & \left|\frac{t^{\alpha}(1-t)}{(1-\eta) \eta^{\alpha}}\right|\left[\int_{0}^{\eta} \frac{(\eta-u)^{\alpha-1}}{\Gamma(\alpha)}\left(\int_{0}^{u} \frac{(u-s)^{\beta-1}}{\Gamma(\beta)}\left|v_{1}(s)-v_{2}(s)\right| d s+\lambda|| x(u)-\bar{x}(u) \mid\right) d u\right] .
\end{aligned}
$$

Hence,

$$
\left\|h_{1}-h_{2}\right\|_{\infty} \leq\left[\frac{\|m\|_{\infty}}{\Gamma(\alpha+\beta+1)}\left(1+A\left(\eta^{\alpha+1}+\eta^{\beta}\right)\right)+\frac{|\lambda|}{\Gamma(\alpha+1)}\left(1+A\left(1+\eta^{\alpha+1}\right)\right)\right]\|x-\bar{x}\|_{\infty} .
$$

Analogously, interchanging the roles of $x$ and $\bar{x}$, we obtain

$$
\begin{aligned}
& H_{d}(N(x), N(\bar{x})) \leq \gamma\|x-\bar{x}\|_{\infty} \\
& \leq\left[\frac{\|m\|_{\infty}}{\Gamma(\alpha+\beta+1)}\left(1+A\left(\eta^{\alpha+1}+\eta^{\beta}\right)\right)+\frac{|\lambda|}{\Gamma(\alpha+1)}\left(1+A\left(1+\eta^{\alpha+1}\right)\right)\right]\|x-\bar{x}\|_{\infty} .
\end{aligned}
$$


Since $N$ is a contraction, it follows by Lemma 11.2.4 that $N$ has a fixed point $x$ which is a solution of (1.1). This completes the proof. $\square$

Example 3.1 Consider the problem

$$
\left\{\begin{array}{l}
{ }^{c} D^{3 / 2}\left({ }^{c} D^{1 / 2}+\frac{1}{3}\right) x(t) \in F(t, x(t)), \quad 0<t<1, \\
x(0)=0, \quad x\left(\frac{1}{3}\right)=0, \quad x(1)=0,
\end{array}\right.
$$

where $F:[0,1] \times \mathbb{R} \rightarrow \mathcal{P}(\mathbb{R})$ is a multivalued map given by

$$
x \rightarrow F(t, x)=\left[\frac{1}{4} \frac{x^{5}}{x^{5}+3}+\frac{1}{8}(t+1), \frac{1}{4} \sin x+\frac{1}{4}(t+1)\right] .
$$

For $f \in F$, we have

$$
|f| \leq \max \left[\frac{1}{4} \frac{x^{5}}{x^{5}+3}+\frac{1}{8}(t+1), \frac{1}{4} \sin x+\frac{1}{4}(t+1)\right] \leq \frac{3}{4} .
$$

Thus,

$$
\|F(t, x)\|_{\mathcal{P}}:=\sup \{|y|: y \in F(t, x)\} \leq p(t) \psi\left(\|x\|_{\infty}\right), \quad x \in \mathbb{R},
$$

with $p(t)=1, \psi\left(\|x\|_{\infty}\right)=3 / 4$. Further, using the condition

$$
\frac{\left[\Gamma(\alpha+1)-|\lambda|\left(1+A\left(1+\eta^{\alpha+1}\right)\right)\right] \Gamma(\alpha+\beta+1)\|x\|_{\infty}}{\Gamma(\alpha+1)\left[1+A\left(\eta^{\alpha+1}+\eta^{\beta}\right)\right]\|p\|_{\infty} \psi\left(\|x\|_{\infty}\right)}>1,
$$

we find that

$$
M>M_{1} \approx 1.255966 .
$$

Clearly, all the conditions of Theorem 3.1 are satisfied. So there exists at least one solution of the problem $(3.3)$ on $[0,1]$.

\section{Acknowledgements}

This project was funded by the Deanship of Scientific Research (DSR), King Abdulaziz University, Jeddah, under grant no. $07 / 31 / G$ r. The authors, therefore, acknowledge with thanks DSR technical and financial support. The authors also thank the referees for their useful comments.

\section{Author details}

${ }^{1}$ Department of Mathematics, Faculty of Science, King Abdulaziz University, P. O. Box 80203, Jeddah 21589, Saudi Arabia ${ }^{2}$ Departamento de Análisis Matemisático, Facultad de Matemáticas, Universidad de Santiago de Compostela, 15782, Santiago de Compostela, Spain

\section{Authors' contributions}

Each of the authors, BA, JJN, and AA contributed to each part of this work equally and read and approved the final version of the manuscript.

\section{Competing interests}

The authors declare that they have no competing interests.

Received: 25 January 2012 Accepted: 8 May 2012 Published: 8 May 2012

\section{References}

1. Kilbas, AA, Srivastava, HM, Trujillo, JJ: Theory and Applications of Fractional Differential Equations. In North-Holland Mathematics Studies, vol. vol. 204,Elsevier Science B.V., Amsterdam (2006)

2. Lakshmikantham, V, Leela, S, Devi, JV: Theory of Fractional Dynamic Systems. Cambridge Academic Publishers, Cambridge (2009) 
3. Sabatier J, Agrawal OP, Machado JAT (eds.): Advances in Fractional Calculus: Theoretical Developments and Applications in Physics and Engineering. Springer, Dordrecht (2007)

4. Baleanu, D, Diethelm, K, Scalas, E, Trujillo, JJ: Fractional Calculus Models and Numerical Methods Series on Complexity, Nonlinearity and Chaos. World Scientific, Boston (2012)

5. Ahmad, B, Otero-Espinar, V: Existence of solutions for fractional differential inclusions with anti-periodic boundary conditions. Bound Value Probl 2009, 11 (2009). (Article ID 625347)

6. Agarwal, RP, Belmekki, M, Benchohra, M: A survey on semilinear differential equations and inclusions involving Riemann-Liouville fractional derivative. Adv Diff Equ 2009, 47 (2009). (Article ID 981728)

7. Chang, YK, Nieto, JJ: Some new existence results for fractional differential inclusions with boundary conditions. Math Comput Model. 49, 605-609 (2009). doi:10.1016/j.mcm.2008.03.014

8. Agarwal, RP, Andrade, B, Cuevas, C: On type of peri-odicity and ergodicity to a class of fractional order differential equations. Adv Diff Equ 2010, 25 (2010). (Article ID 179750)

9. Bai, B: On positive solutions of a nonlocal fractional boundary value problem. Nonlinear Anal. 72, 916-924 (2010). doi:10.1016/j.na.2009.07.033

10. Zhang, S: Positive solutions to singular boundary value problem for nonlinear fractional differential equation. Comput Math Appl. 59, 1300-1309 (2010). doi:10.1016/j.camwa.2009.06.034

11. Balachandran, K, Trujillo, Jj: The nonlocal Cauchy problem for nonlinear fractional integrodifferential equations in Banach spaces. Nonlinear Anal. 72, 4587-4593 (2010). doi:10.1016/.na.2010.02.035

12. Agarwal, RP, Andrade, B, Cuevas, C: Weighted pseudo-almost periodic solutions of a class of semilinear fractional differential equations. Nonlinear Anal Real World Appl. 11, 3532-3554 (2010). doi:10.1016/j.nonrwa.2010.01.002

13. Ahmad, B, Sivasundaram, S: On four-point nonlocal boundary value problems of nonlinear integro-differential equations of fractional order. Appl Math Comput. 217, 480-487 (2010). doi:10.1016/j.amc.2010.05.080

14. Baleanu, D, Mustafa, OG, Agarwal, RP: An existence result for a superlinear fractional differential equation. Appl Math Lett. 23, 1129-1132 (2010). doi:10.1016/j.aml.2010.04.049

15. Baleanu, D, Mustafa, OG, Agarwal, RP: On the solution set for a class of sequential fractional differential equations. J Phys A 43(38):7 (2010). (Article ID 385209)

16. Cernea, A: On the existence of solutions for nonconvex fractional hyperbolic differential inclusions. Commun Math Anal. 9(1):109-120 (2010)

17. Ahmad, B: Existence results for fractional differential inclusions with separated boundary conditions. Bull Kor Math Soc. 47, 805-813 (2010). doi:10.4134/BKMS.2010.47.4.805

18. Ahmad, B, Ntouyas, SK: Some existence results for boundary value problems fractional differential inclusions with nonseparated boundary conditions. Electron J Qual Theory Diff Equ. 71, 1-17 (2010)

19. Ahmad, B, Ntouyas, SK: A four-point nonlocal integral boundary value problem for fractional differential equations of arbitrary order. Electron J Qual Theory Diff Equ. 22, 1-15 (2011)

20. Ahmad, B, Agarwal, RP: On nonlocal fractional boundary value problems. Dyn Contin Discr Impuls Syst Ser A Math Anal. 18(2011):535-544 (2011)

21. Ahmad, B, Ntouyas, SK: Boundary value problems for q-difference inclusions. Abstr Appl Anal 2011, 15 (2011). (Article ID 292860)

22. Agarwal, RP, Ahmad, B: Existence theory for anti-periodic boundary value problems of fractional differential equations and inclusions. Comput Math Appl. 62, 1200-1214 (2011). doi:10.1016/j.camwa.2011.03.001

23. Ahmad, B, Nieto, JJ, Pimentel, J: Some boundary value problems of fractional differential equations and inclusions. Comput Math Appl. 62, 1238-1250 (2011). doi:10.1016/j.camwa.2011.02.035

24. Ahmad, B, Nieto, JJ: Riemann-Liouville fractional integro-differential equations with fractional nonlocal integral boundary conditions. Bound Value Probl. 2011(36):9 (2011)

25. Ahmad, B, Alsaedi, A: Existence and uniqueness of solutions for coupled systems of higher-order nonlinear fractional differential equations. Fixed Point Theory Appl 2010, 17 (2011). (Article ID 364560)

26. Santos, JPC, Cuevas, C, Arjunan, M: Existence results for a fractional neutral integro-differential equations with statedependent delay. Comput Math Appl. 62, 275-1283 (2011)

27. Wax N (ed.): Selected Papers on Noise and Stochastic Processes. Dover, New York (1954)

28. Mazo, R: Brownian Motion: Fluctuations, Dynamics and Applications. Oxford University Press, Oxford (2002)

29. Coffey, WT, Kalmykov, YP, Waldron, JT: The Langevin Equation. World Scientific, Singapore, 2 (2004)

30. Wang, KG: Long-time-correlation effects and biased anomalous diffusion. Phys Rev A. 45, 833-837 (1992). doi:10.1103/ PhysRevA.45.833

31. Porra, JM, Wang, KG, Masoliver, J: Generalized Langevin equations: anomalous diffusion and probability distributions. Phys Rev E. 53, 5872-5881 (1996). doi:10.1103/PhysRevE.53.5872

32. Wang, KG, Tokuyama, M: Nonequilibrium statistical description of anomalous diffusion. Phys A. 265, 341-351 (1999). doi:10.1016/50378-4371(98)00644-X

33. Lutz, E: Fractional Langevin equation. Phys Rev E. 64, 051106 (2001)

34. Fa, KS: Generalized Langevin equation with fractional derivative and long-time correlation function. Phys Rev. 73, 061104 (2006)

35. Fa, KS: Fractional Langevin equation and Riemann-Liouville fractional derivative. Eur Phys J E. 24, 139-143 (2007). doi:10.1140/epje/i2007-10224-2

36. Kobolev, V, Romanov, E: Fractional Langevin equation to describe anomalous diffusion. Prog Theo Phys Suppl. 139 470-476 (2000)

37. Lim, SC, Muniandy, SV: Self-similar Gaussian processes for modeling anomalous diffusion. Phys Rev E. 66, 021114 (2002)

38. Picozzi, S, West, B: Fractional Langevin model of memory in financial markets. Phys Rev E. 66, 046118 (2002)

39. Lim, SC, Li, M, Teo, LP: Locally self-similar fractional oscillator processes. Fluc Noise Lett. 7, 169-179 (2007). doi:10.1142/ S0219477507003817

40. Lim, SC, Li, M, Teo, LP: Langevin equation with two fractional orders. Phys Lett A. 372, 6309-6320 (2008). doi:10.1016/j. physleta.2008.08.045

41. Lim, SC, Teo, LP: The fractional oscillator process with two indices. J Phys A Math Theory. 42, 065208 (2009). doi:10.1088/1751-8113/42/6/065208 
42. Ahmad, B, Nieto, Jj: Solvability of nonlinear Langevin equation involving two fractional orders with Dirichlet boundary conditions. Int J Diff Equ 2010, 2 (2010). (Article ID 649486)

43. Uranagase, M, Munakata, T: Generalized Langevin equation revisited: mechanical random force and self-consistent structure. J Phys A Math Theor 43, 11 (2010). (Article ID 455003)

44. Denisov, SI, Kantz, H, Hänggi, P: Langevin equation with super-heavy-tailed noise. J Phys A: Math Theor 43, 10 (2010). (Article ID 285004)

45. Lozinski, A, Owens, RG, Phillips, TN: The Langevin and Fokker-Planck Equations in Polymer Rheology. In Handbook of Numerical Analysis, vol. vol. 16, pp. 211-303.Elsevier, Amsterdam (2011)

46. Lizana, L, Ambjörnsson, T, Taloni, A, Barkai, E, Lomholt, MA: Foundation of fractional Langevin equation: harmonization of a many-body problem. Phys Rev E 81, 8 (2010). (Article ID 051118)

47. Ahmad, B, Nieto, JJ, Alsaedi, A, El-Shahed, M: A study of nonlinear Langevin equation involving two fractional orders in different intervals. Nonlinear Anal Real World Appl. 13, 599-606 (2012). doi:10.1016/.jnonrwa.2011.07.052

48. Deimling, K: Multivalued Differential Equations. Walter De Gruyter, Berlin/New York (1992)

49. Hu, S, Papageorgiou, N: Handbook of Multivalued Analysis. Kluwer, Dordrechtvol. I: Theory (1997)

50. Kisielewicz, M: Differential Inclusions and Optimal Control. Kluwer, Dordrecht (1991)

51. Lasota, A, Opial, Z: An application of the Kakutani-Ky Fan theorem in the theory of ordinary differential equations. Bull Acad Polon Sci Ser Sci Math Astronom Phys. 13, 781-786 (1965)

52. Granas, A, Dugundji, J: Fixed Point Theory. Springer-Verlag, New York (2005)

53. Bressan, A, Colombo, G: Extensions and selections of maps with decomposable values. Studia Math. 90, 69-86 (1988)

54. Covitz, H, SNadler, SB Jr: Multivalued contraction mappings in generalized metric spaces. Israel J Math. 8, 5-11 (1970). doi:10.1007/BF02771543

55. Yu, L, Liang, M: Convergence of iterative sequences for fixed point and variational inclusion problems. Fixed Point Theory Appl 2011, 15 (2011). (Article ID 368137). doi:10.1186/1687-1812-2011-15

56. He, Z, Du, WS, Lin, IJ: The existence of fixed points for new nonlinear multivalued maps and their applications. Fixed Point Theory Appl. 2011, 84 (2011). doi:10.1186/1687-1812-2011-84

57. Castaing, C, Valadier, M: Convex Analysis and Measurable Multifunctions. Lecture Notes in Mathematics 580. SpringerVerlag, Berlin/Heidelberg/New York (1977)

doi:10.1186/1687-1847-2012-54

Cite this article as: Ahmad et al:: A nonlocal three-point inclusion problem of Langevin equation with two different fractional orders. Advances in Difference Equations 2012 2012:54.

\section{Submit your manuscript to a SpringerOpen ${ }^{\circ}$ journal and benefit from:}

Convenient online submission

- Rigorous peer review

- Immediate publication on acceptance

- Open access: articles freely available online

- High visibility within the field

- Retaining the copyright to your article

Submit your next manuscript at $\gg$ springeropen.com 